\section{Thromboembolic ST elevation myocardial infarction due to a large coronary aneurysm: Role of apixaban}

\author{
Stefania Cherubini* (D), Alessandro Sciahbasi** (D), Maria Cera** (D), \\ Silvio Fedele** (D), Giuseppe Ferraiuolo** (D), Andrea Ciolli* (D) \\ Departments of *UOSD Emergency Cardiology, and **Interventional \\ Cardiology, UOC Cardiologia, Sandro Pertini Hospital; Rome-Italy
}

\section{Introduction}

Coronary artery aneurysms (CAAs) are generally defined as a localized coronary dilatation exceeding the normal adjacent diameter of the coronary artery by more than 1.5 fold, which is considered as a reference point (1). The prevalence ranges from $1.4 \%$ to $4.9 \%$ (2) on diagnostic coronary angiography, but aneurysms larger than $8 \mathrm{~mm}$ are less frequently described (3). Most patients with CAA are asymptomatic, and the aneurysm is incidentally detected during coronary angiography. Sometimes aneurysm is associated with an acute coronary syndrome due to thrombotic occlusion of the aneurysm or distal embolization. Therapy of patients with large aneurysm associated with acute coronary syndrome is a management dilemma, particularly the choice of antithrombotic therapy because large studies and dedicated guidelines are lacking.

In this case report, we describe a patient with ST elevation myocardial infarction due to large coronary aneurysm and distal thromboembolic vessel occlusion treated with balloon coronary angioplasty and dual antithrombotic therapy with clopidogrel and apixaban.

\section{Case Report}

A 40-year-old woman was admitted to our Emergency Department for typical chest pain and dyspnea in the absence of known cardiologic risk factors. The electrocardiogram showed ST elevation in leads II, III, and augmented vector foot (aVF), and an emergency coronary angiography was performed for acute ST elevation myocardial infarction. The angiography revealed a large fusiform coronary aneurysm (8 $\mathrm{mm}$ in diameter) of the proximal tract of the right coronary artery and thrombotic occlusion of the distal vessel (Fig. 1a). There were no significant lesions in the left coronary artery (Fig. 1b). In the same setting, the vessel was recanalized with plain balloon angioplasty with a $2.5 \times 20 \mathrm{~mm}$ balloon (Emerge, Boston Scientific) inflated at 10 atmosphere without evidence of residual atherosclerosis (Fig. 1c). Peri-procedural antithrombotic therapy included aspirin (250 mg intravenous), clopidogrel (300 $\mathrm{mg}$ ), and unfractionated heparin (100 units $/ \mathrm{kg}$ ). Two days after the procedure, aspirin therapy was stopped, clopidogrel $75 \mathrm{mg}$ per day was maintained, and apixaban $5 \mathrm{mg}$ twice per day was added. An ultrasonic echocardiogram showed a moderate reduction of ejection fraction (EF) to $40 \%$ with inferior akinesia. The patient did not report a history of previous Kawasaki disease or other infectious diseases (such as HIV, syphilis, or Lyme disease), and hematological screening for autoimmune disease was negative. A rheumatologic evaluation excluded the presence of connective tissue disorders. During the hospitalization, there were no complications, and the patient was discharged after 7 days with clopidogrel and apixaban. At the 1-year follow-up, the patient was asymptomatic, the echocardiogram showed an improvement in $\mathrm{EF}$ to $52 \%$, and a multidetector computed tomography coronary angiography confirmed the patency of the right coronary artery (Fig. 2).

\section{Discussion}

The ideal pharmacological therapy of CAA is not well-defined because of lack of evidence. The aneurysmal dilatation provides a nidus for acute thrombus formation through a more sluggish flow and stasis promoting platelet activation and thrombus formation with possible distal embolization and consequent acute coronary syndrome. In this context, the use of oral anticoagulant therapy has been proposed (4) to reduce the incidence of CAA thrombosis, but its adoption is controversial, particularly in the

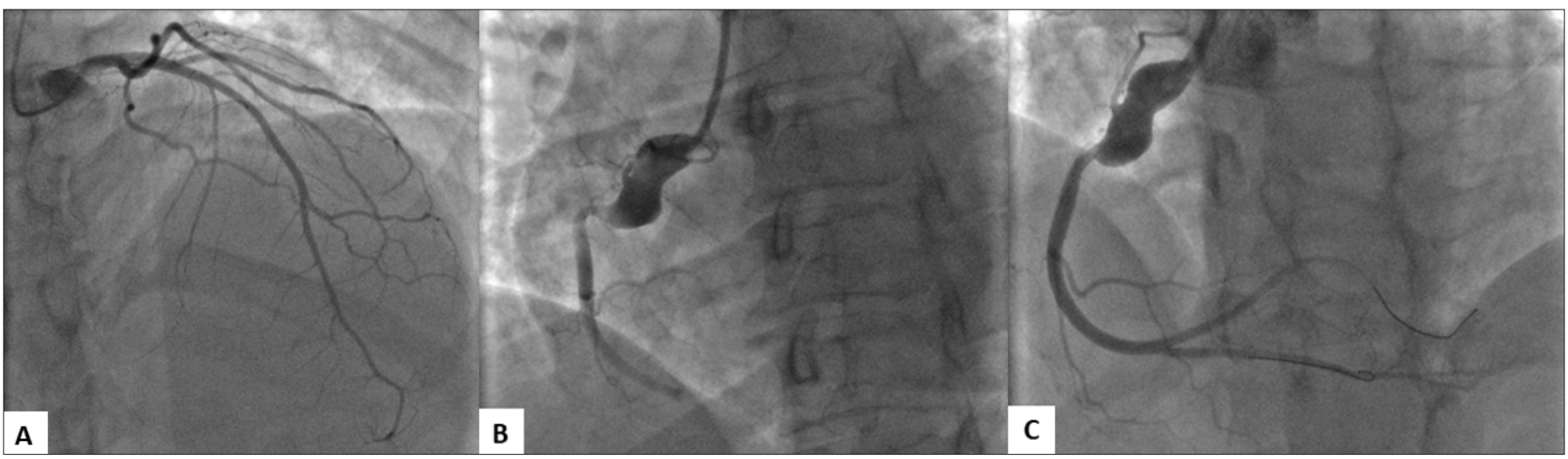

Figure 1. Coronary angiography before and after percutaneous coronary procedure. (a) Baseline left coronary artery. (b) Baseline right coronary artery showing the aneurysm and the embolic vessel occlusion. (c) Final result after plain balloon coronary angioplasty 

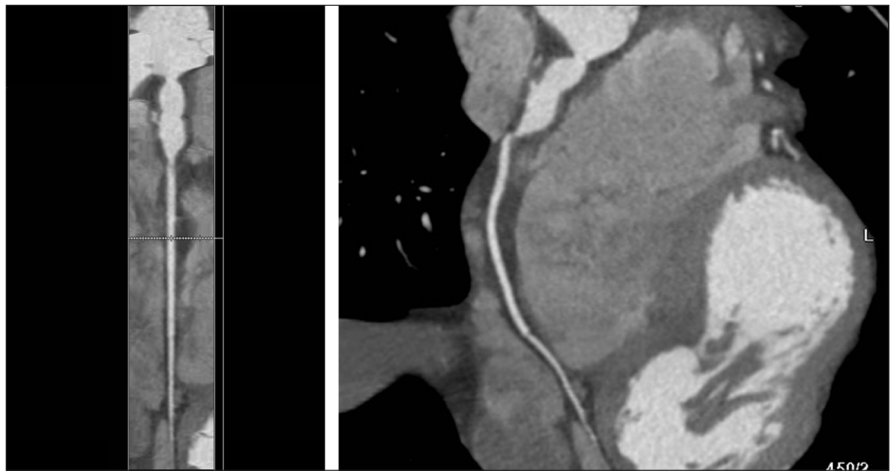

Figure 2. Multidetector computed tomography coronary angiography. The exam confirmed the patency of the right coronary artery at follow-up

setting of acute coronary syndromes that also require antiplatelet therapy. In the largest registry of CAA [the coronary artery aneurysms registry (CAAR)] (5), most of the patients were discharged without anticoagulant therapy and only a minority were discharged with a warfarin-based therapy. However, in the subgroup of patients with large CAA, the rate of anticoagulant use was higher. In the CAAR, the use of a warfarin-based therapy decreased the composite endpoint of unstable angina, myocardial infarction, and aneurysm thrombosis confirming the value of anticoagulant therapy in this setting of patients. Over the past years, direct oral anticoagulants (apixaban, edoxaban, rivaroxaban, and dabigatran) have shown greater efficacy and safety than warfarin for the prevention of thromboembolic events in patients with atrial fibrillation or venous thromboembolism. To date, little data are available for the use of these direct oral anticoagulants in patients with CAA. Chen et al. (6) recently presented a single patient with giant left CAA treated with a combination therapy of rivaroxaban and clopidogrel, but the follow-up was limited to 2 months owing to the need of heart transplantation. In another case report (7), a 75-year-old patient with inferior ST elevation myocardial infarction and CAA was treated with a combination of aspirin, prasugrel, and very low-dose rivaroxaban (2.5 mg twice a day). In another 2 studies, Korosoglou et al. (8) documented the use of rivaroxaban $15 \mathrm{mg}$ with aspirin and clopidogrel in a 73-year-old female with acute coronary syndrome and coronary aneurysm, and Yan et al. (9) documented the use of rivaroxaban $20 \mathrm{mg}$ and aspirin in a 33-year-old man with acute myocardial infarction and coronary aneurysm but no evidence of vessel occlusion. Finally, Esenboğa et al. (10) demonstrated short-term use of apixaban in 2 patients with non-ST elevation myocardial infarction.

To the best of our knowledge, this is the first case report on the possible use of apixaban for CAA to prevent thrombotic events leading to acute ST elevation myocardial infarction. In our patient, the combination of apixaban and clopidogrel avoided further thrombotic events maintaining the patency of the coronary artery at 1-year follow-up, and the anticoagulant therapy has been continued.

\section{Conclusion}

Our report supports the possible use of apixaban over warfarin in the antithrombotic treatment of patients with CAA and myocardial infarction. More reports are needed to confirm the efficacy of this pharmacological strategy in patients with large CAA.

Informed consent: Patient informed consent was waived owing to the retrospective nature of this case report.

\section{References}

1. Núñez-Gil IJ, Terol B, Feltes G, Nombela-Franco L, Salinas P, Escaned $\mathrm{J}$, et al. Coronary aneurysms in the acute patient: Incidence, characterization and long-term management results. Cardiovasc Revasc Med 2018; 19: 589-96.

2. Cohen P, O'Gara PT. Coronary artery aneurysms: a review of the natural history, pathophysiology, and management. Cardiol Rev 2008; 16: 301-4.

3. Kato $H$, Sugimura $T$, Akagi $T$, Sato $N$, Hashino $K$, Maeno $Y$, et al. Long-term consequences of Kawasaki disease. A 10- to 21-year follow-up study of 594 patients. Circulation 1996; 94: 1379-85.

4. Denby KJ, Clark DE, Markham LW. Management of Kawasaki disease in adults. Heart 2017; 103: 1760-9.

5. D’Ascenzo F, Saglietto A, Ramakrishna H, Andreis A, Jiménez-Mazuecos JM, Nombela-Franco L, et al.; CAAR Investigators. Usefulness of oral anticoagulation in patients with coronary aneurysms: Insights from the CAAR registry. Catheter Cardiovasc Interv 2020 Sep 9. doi: 10.1002/ccd.29243. [Epub ahead of print]

6. Chen T, Li J, Xu Q, Li X, Lv O, Wu H. Antithrombotic Therapy of a Young Adult with Giant Left Main Coronary Artery Aneurysm. Int Heart J 2020; 61: 601-5.

7. Bath A, Shaikh F, Kalavakunta JK. Coronary artery aneurysm presenting as STEMI. BMJ Case Rep 2019; 12: e231013.

8. Korosoglou G, Eisele T, Nunninger P, Giusca S. Mechanical and pharmacologic treatment in a patient with a coronary artery aneurysm and acute coronary syndrome. Coron Artery Dis 2017; 28: $358-60$.

9. Yan 0 , Ning L, Jian Y, Yang W, Yuan Q, Du Z. Could the Novel Oral Anticoagulants Be Used for Coronary Artery Aneurysm? Case Rep Med 2020; 2020: 5073814.

10. Esenboğa K, Şahin E, Özyüncü N, Tan TS, Atmaca Y. Apixaban for massive intracoronary thrombosis: A case series. Anatol J Cardiol 2021; 25: 661-4.

Address for Correspondence: Alessandro Sciahbasi, MD, Department of Interventional Cardiology, UOC Cardiologia,

Sandro Pertini Hospital; Rome-Italy

Phone: +39-06-41433870

E-mail: asciahbasi@gmail.com

(C) Copyright 2021 by Turkish Society of Cardiology -

Available online at www.anatoljcardiol.com

D0I:10.5152/AnatolJCardiol.2021.128 